\title{
Differentiated Service in IP Networks Using Voice and Data Applications
}

\author{
Marlenne Angulo and Marco A.Turrubiartes \\ Universidad Autónoma de Baja California, México
}

\section{mangulo@uabc.mx $\underline{\text { mturrubiartes@uabc.mx }}$}

\begin{abstract}
One of the options that telephonic systems have to optimize the required bandwidth is to migrate to statistical multiplexing systems. These multiplexing schemes also allow the convergence with current data communications systems.

This work is based in the interconnection of telephonic systems through the data network using digitalization and voice compression techniques.

The optimization of the resources is achieved using the voice compression standard of $8 \mathrm{kbps}$ instead of the $64 \mathrm{kbps}$ standard, thus, introducing differentiated services in IP networks. The performance of the voice packages is improved in terms of delay and package losses.

The results presented were obtained by means of computer simulations using COMNET. The merit of this work is the usage of real telephonic traffic information obtained from the call recorders of the $\mathrm{UABC}$, the information was acquired for several months, which allowed the consideration of effects such as rush hours and variable call duration.
\end{abstract}

The performance of the system was evaluated, obtaining very satisfactory results in terms of resources utilization.

Keywords : Voice over IP networks, Differentiated Services, Quality of service

\section{Introduction}

The IP networks globalization and the increase in the diversity of applications and services, demands that they become capable of manage different quality of service requirements (Angulo et. al, 2000).

Unlike the voice network, data networks use information packets and packets switching devices to transmit the information, this way, they optimize the usage of the information transmission capabilities.

This article is based in the interconnection of the telephonic systems by means of E\&M signaling through the data network, using digitalization and voice compression techniques and international standards.

This paper is organized as follows: the second section gives a brief introduction to the telephonic system, in third section Fundamental concepts of IP networks that offer differentiated services are de-

Material published as part of these proceedings, either on-line or in print, is copyrighted by Informing Science. Permission to make digital or paper copy of part or all of these works for personal or classroom use is granted without fee provided that the copies are not made or distributed for profit or commercial advantage AND that copies 1) bear this notice in full and 2) give the full citation on the first page. It is permissible to abstract these works so long as credit is given. To copy in all other cases or to republish or to post on a server or to redistribute to lists requires specific permission from the publisher at Publisher@InformingScience.org scribed, the next section contents the simulation considerations, followed by the results presentation and analysis. Finally, the conclusion is presented. 


\section{Telephonic system}

Two States Markovian Model for Individual Voice Sources.

The typical behavior of packed voice sources is as follows: (Daigle et. al., 1986). A source is said to be "active" when a user is literally speaking, during this period, the voice source generates packets of fixed length at regular time intervals. Afterwards, comes a silence period during which the source changes it state to "inactive" and no packets are produced. When we identify these two states of the process, it is possible to model the voice with Markovian chains since they allow us to characterize a discrete variable that changes in the time domain through its states transition probabilities.

A packet burst from a voice source is being model by arrivals at fixed intervals with a $\mathrm{T}$ $m$ secs duration, and by a silence period during which there is no arrival (figure 1). According to Heffes [4], a voice source is modeled by the next equation:

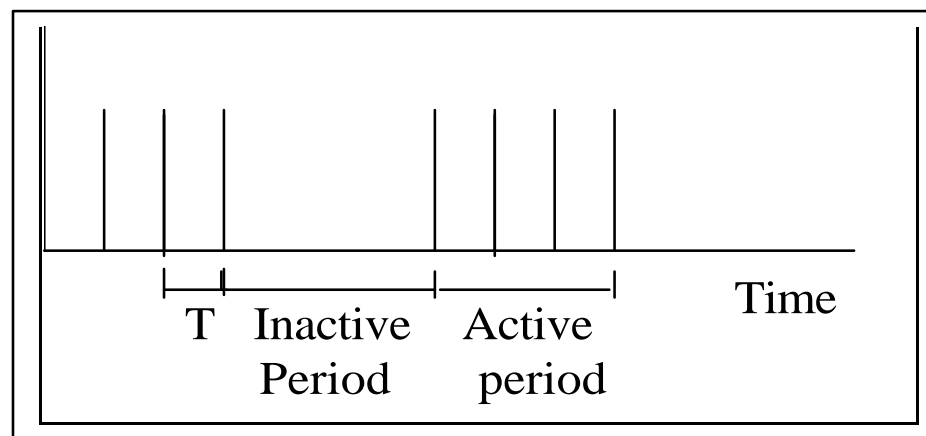

Figure 1. Packetized voice source

$$
F(t)=\left[(1-\alpha T)+\alpha T\left(1-e^{-\beta(t-T)}\right)\right] U(t-T)
$$

Where $U(t)$ is the singled step function, $\alpha$ is the average value of the active period, $\beta$ is the average value of the silence period; both periods are described by exponential distribution functions, the inter arrival rate is of $1 / \alpha \mathrm{T}$ packets, and corresponds to a geometric distribution.

\section{Differentiated Services}

Differentiated Services is an architecture that proposes scalability by means of aggregated traffic classification states. They are implemented in the network layer where the category of a packet is marked in one or more fields of the IP layer header.

The differentiated services can be implemented under two modes: Expedited Forward and Assured Forward.

The expedited forward mode was designed to support connections with low losses, delays and jitter. This mode shows a behavior similar to the virtual dedicated lines between two final points with a peak bandwidth.

The assured forward mode defines fours related service classes, where each one supports three discharge priority levels. When congestion occurs in the router, the packets with higher discharge precedence are discharged first, followed by the lower discharge precedence packets. The four assured forward classes do not have the values of bandwidth and delay defined.

The architecture proposed by RFC 2475 (Blake et. al., 1998), defines that the differentiated services must classify individual micro flows at the gate of the network in one of four classes of services. The admission to the network is based in the analysis of one or more fields in the packet. The packet is marked (changing some header bits of fields) to specify that it belong to certain kind of service class, then, it is admitted in the network. 


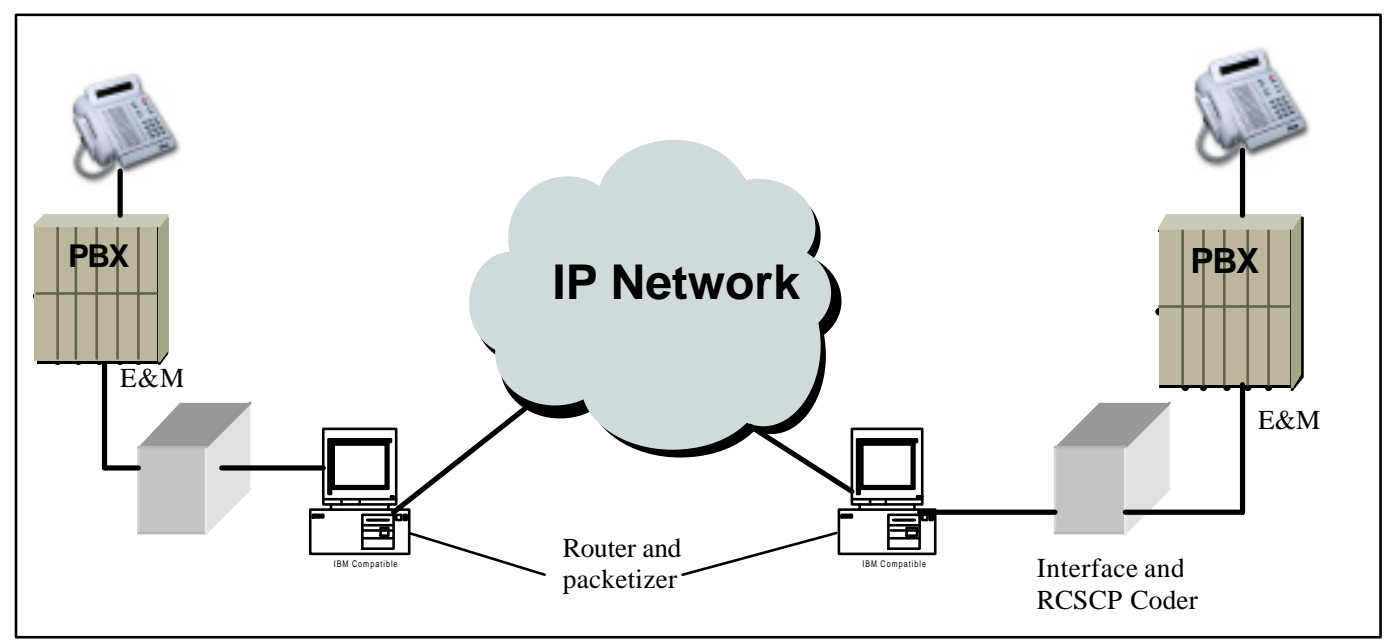

Figure 2.- Implemented scheme of voice over IP

\section{Simulation considerations}

In figure 2, we can observe a voice outline through IP network. This article does not consider the E\&M signaling process, focusing in the analysis of router and packetizer. The codification standards used are the G729A (CS-CELP) and the G732 (ITU G729, 1996) and (ITU G729a, 1986).

In the packetizing process the delay of the process must be considered as well as the size of the generated packet, including the headers of the protocols required for the information transport. In the equation 2, the load due to one single voice source and a sample of the voice codifier in the packet are represented.

Load $[b p s]=\left[M_{b}+E t_{b}+I P_{b}+U D P_{b}+R T P_{b}\right] /(M t)$

Where $\mathrm{Mb}$ represent the sample size expressed in bits, $\mathrm{Et}_{\mathrm{b}}, \mathrm{Ip}_{\mathrm{b}}, \mathrm{UDP}_{\mathrm{b}}, \mathrm{RTP}_{\mathrm{b}}$ represents the Ethernet, IP, UDP and RTP headers respectively, and Mt represents the codifier sample generation interval expressed in sec.

$$
\text { Load }[b p s]=\left[n M_{b}+\left(E t_{b}+I P_{b}+U D P_{b}+R T P_{b}\right)\right] /(n M t)
$$

In this equation we can observe that the load of the network decreases as $n$ increases, however there are other parameters considered in this simulation such are packet losses, maximum delay and delay variations. Besides, it is necessary to take into account that a big part of the IP ne twork traffic is not voice, but data, so data traffic such as www represents outbursts and increases the stowage in the interconne ction devices.

Differentiated Services are used to give priority to voice packets over data packets inside the routers; discarding the usage of upper layer protocols such are RTP/ RTCP.

The IP network was simulated using COMNET (figure 3), there, the call generators, background traffic generators, codifiers, packeters and routers where simulated.

The IP network service the packets depending of their service priority, where the voice packets have a service rate double the remaining packets service rate. The primary function of an IP router is to direct the packet received through an input port to an output port depending on the destiny address of the packet and the routing table. To simulation effects all the traffic converges in the same output port. 


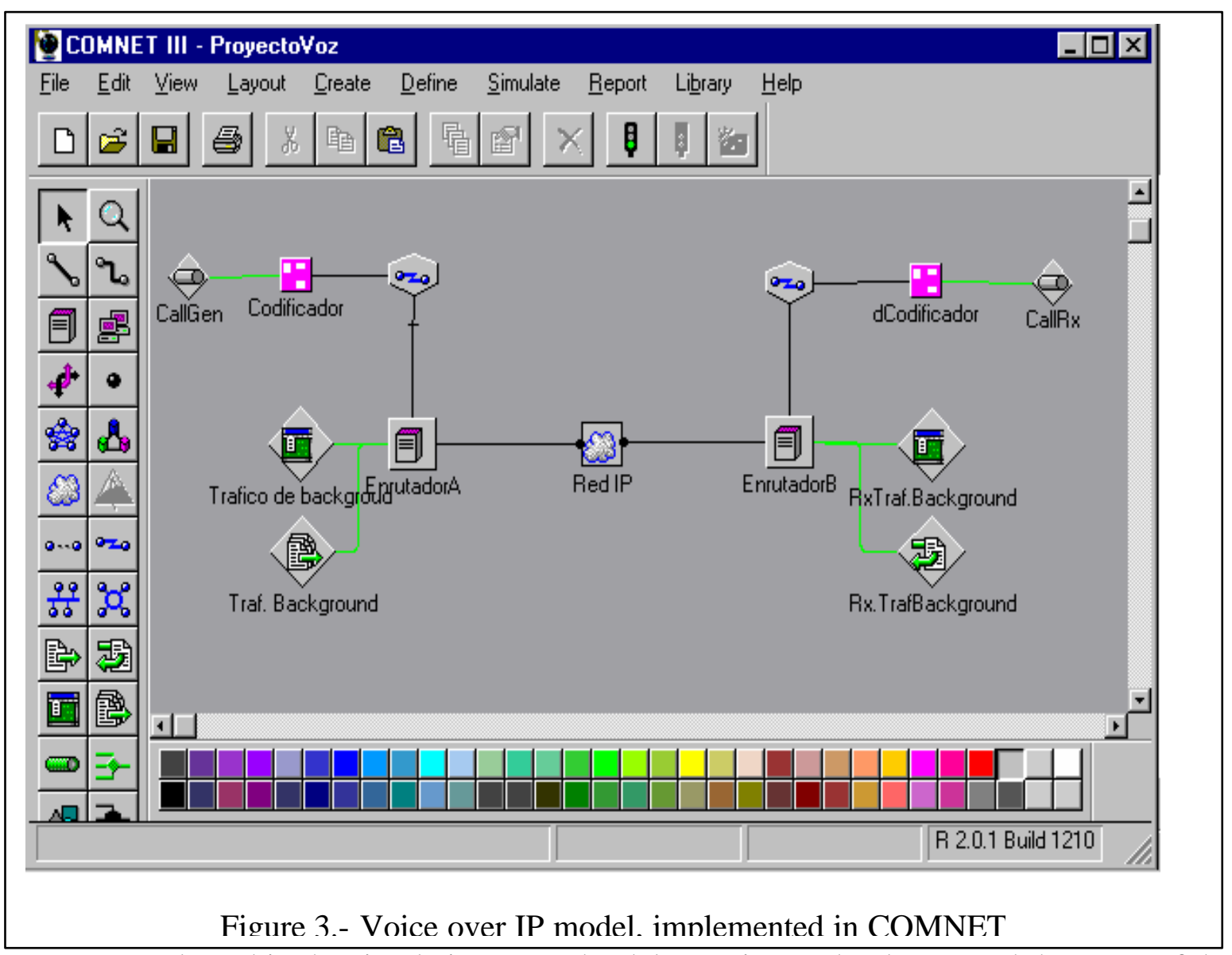

The parameters evaluated in the simulation were the delay, voice packet losses and the usage of the routers links. Although the main goal of this article was the optimization of the bandwidth in the trunk links, it was verified that the outline didn't degrade one of the most significant parameters of the voice service quality: the delay. Figure 4 shows us a graphic of the obtained delay in voice packets with an 8 kbps codification. The voice packet losses average during 500 seconds of simulation was $0.7 \%$, which is below the $1 \%$ limit. The used outline with an 8 kbps codification shows an effective reduction of $30 \%$ of the bandwidth in the transmission line respect to $64 \mathrm{kbps}$.

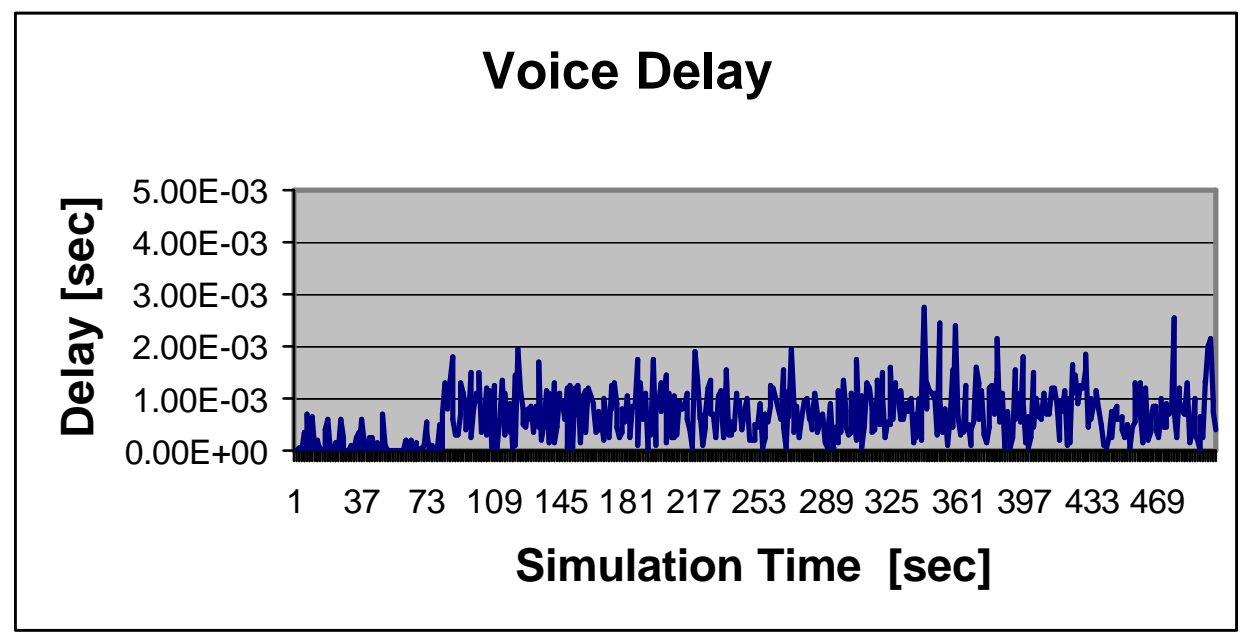

Figure 4.- Voice delay obtained by simulation with 8kbps compression 


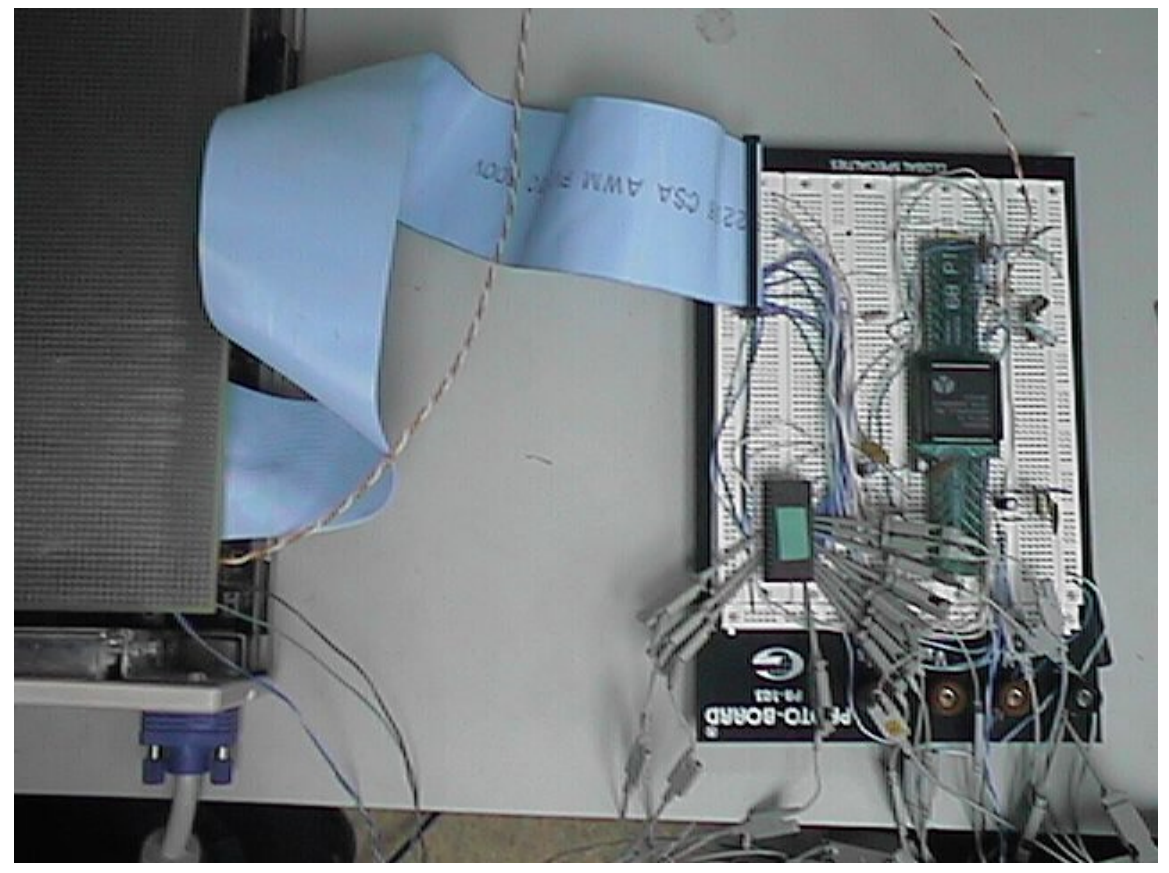

Figure 5.- Voice coder circuit under test

Figure 5 it is a photo of the testing montage of the RCSCP voice codifier circuit (from Conexant Semiconductor Systems), where, by means of a logic state analyzer, we were able to verify its performance.

\section{Conclusions}

The performance evaluation of the outline used was made doing an effective reduction of $30 \%$ in the bandwidth of the transmission line. The result was very satisfactory in terms of resource utilization, an acceptable voice performance was maintained, where parameters such as packet losses and delay were reviewed.

As a future work, it is considered the implementation in printed circuit of the prototype showed in the testing montage, and to characterize the system as well as introduce in the packetizer the programming necessary to handle RTP/ RTCP protocols.

\section{Acknowledgements}

To the Autonomous University of Baja California through the Engineering Faculty and to DGIP for their support to the project "Voice over IP networks, study case: UABC"

\section{References}

Angulo, M., Gallardo, J., Makrakis, D. (2000). Dynamic Bandwidth Allocation Schemes for a Differentiated-Service-

Capable Internet Boundary Node. International Conference on Telecommunication, Acapulco México.

Blake, S., Black, D., Carlson, M. (1998). An architecture for Differentiated Services. Internet Engineering Task Force, RFC 2475 .

Daigle, John N., Langford, Joseph D. (1986). Models for Analysis of Packet Voice communications systems . IEEE Journal on selected areas in communications VOL SAC-4 No.6 p 847-854.

Heffes, Harry, Lucantoni, David. (1986). A markov Modulated Characterization of Packetized Voice and Data Traffic and Related Statistical Multiplexer Performance. IEEE Journal on selected areas in communications Vol SAC-4 No.6 pp $856-867$ 
ITU Reg. G.729 (1996) Coding speech at 8 kbps using Conjugate-Structure Jalgebraic-Code-Excited Linear Prediction CSACELP.

ITU Reg G.729a (1996) Reduced Complexity 8kbps CS-ACELP Speech Codec.

\section{Biographies}

Marco Turrubiartes (IEEE member) is professor at University of Baja California. He has taught data communications and computer network courses in several occasions. Currently he is director of a Voice over IP project at the university. His current research topics are Quality of service in data networks and OnLine education.

Marlenne Angulo (IEEE member) is professor at University of Baja California. Currently she is working in the production and design of didactical material by using new technologies. She is also participating in a university project called a Voice over IP. Her current research topics are multimedia over data networks and Online education. 